\title{
Explosion Characteristics of Hydrogen for CFD Modelling and Simulation of Turbulent Gas Flow
}

\author{
Jan Skř́nský1, ${ }^{1,}$, Jan Koloničný ${ }^{1}$, and Tadeáš Ochodek ${ }^{1}$ \\ ${ }^{1}$ Energy Research Centre, VSB-TU Ostrava, 70833 Ostrava - Poruba, Czech Republic
}

\begin{abstract}
Renewable energies became more and more important in the last years. Hydrogen as a promising energy carrier is a perfect candidate to supply the energy demand of the world. The state of the hydrogen gas (turbulences and point concentrations) has a significant impact on the gas explosion indices. A gas cloud is formed by a partial-pressure method in gas explosion experiments in the spherical $20.0 \cdot 10^{-3} \mathrm{~m}^{3}$ chamber. Gas in the chamber reaches an uniform state beyond in hundreds of ms. The absolute pressure for gas dispersion should be higher than $0.01 \mathrm{MPa}$ for the $\mathrm{H}_{2}$ of concentration larger than 30 vol. \% of fuel. The initial temperature also influences turbulent gas flow before ignition, especially in the case of the gases lighter-than-air.
\end{abstract}

\section{Introduction}

\subsection{Interest}

Results of explosion experiments depend on many different parameters of the investigated process, such as the energy and type of ignition source, size and shape of explosion chamber, initial temperature and composition of the flammable mixture [1]. One way is to study these processes by mathematical computational fluid dynamics (CFD) modelling. CFD has sufficient accuracy and a much higher potential for being optimized if based on currently used experimental methods such as standard $20.0 \cdot 10^{-3} \mathrm{~m}^{3}[2]$.

\subsection{Previous studies}

Explosion indices of individual gases at ambient temperature and atmospheric pressure have been studied systematically. Only limited data are available for hydrogen gas at elevated temperatures and pressures until now. In work [3] has been reported measurement on explosion pressures of hydrogen-air mixtures, at various initial concentrations close to stoichiometric, in closed vessels of different volumes. The results have shown a similar behavior of investigated system. The maximum explosion pressures have been $(7.9 \pm 0.3)$ bar for measurements made in the standard $20.0 \cdot 10^{-3} \mathrm{~m}^{3}$ spherical vessel. Higher deviations from these values are observed, however, when using smaller or larger size vessels, where

\footnotetext{
*Corresponding author: jan.skrinsky@,vsb.cz
} 
radiative and convective heat losses to the walls could be neglected. In [4] have been reported different values of maximum explosion pressures for hydrogen-air mixtures, depending on the volume of the explosion vessel: 8.1 bar in a $120.0 \cdot 10^{-3} \mathrm{~m}^{3}$ explosion vessel. The reported results from $6.0 \cdot 10^{-3}$ are identical to those results obtained in the $20.0 \cdot 10^{-3} \mathrm{~m}^{3}$ and $120.0 \cdot 10^{-3} \mathrm{~m}^{3}$ despite the great difference in volume. The present contribution introduce the explosion pressures of stoichiometric hydrogen - air mixtures measured experimentally in $20.0 \cdot 10^{-3} \mathrm{~m}^{3}$ chamber for various initial temperatures. The temperature influence on the maximum explosion pressure was investigated at atmospheric pressure.

\section{Experiment}

\subsection{Experimental device}

Experiments were done in $20.0 \cdot 10^{-3} \mathrm{~m}^{3}$ chamber (CA 20L) made by OZM Research in accordance with EN 1839 [5]. It is the stainless steel (1.4435) double wall vessel of spherical shape with an internal diameter of $336 \mathrm{~mm}$. The vessel is provided with an opening of an inside diameter of $148 \mathrm{~mm}$. The whole system is schematically introduced at Figure 1.

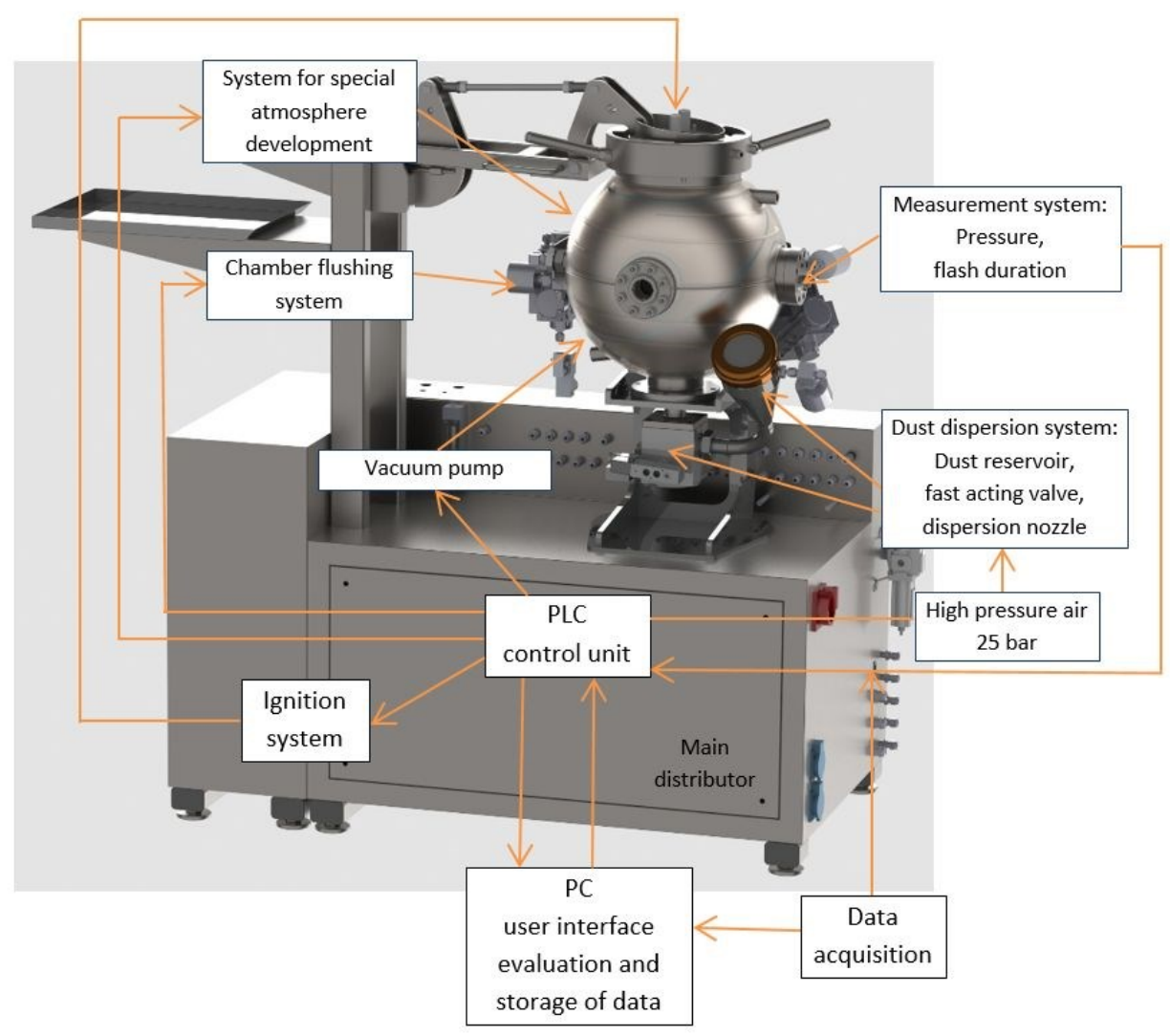

Fig. 1. Schematic introduction of the $20.0 \cdot 10^{-3} \mathrm{~m}^{3}$ chamber system. 
Highly dynamic temperature control system Presto A30 by JULABO is used for heating of the chamber. Permanent spark generator was made in accordance with EN 15967 [6] with Tungsten electrodes with a distance of $6 \mathrm{~mm}$. A pair of piezoelectric pressure sensors by Kistler, type 701A. Pressure range set for the sensors is $2.5 \mathrm{MPa}$ and natural frequency $70 \mathrm{kHz}$. Data are recorded be four-channel data card with a sampling rate $50 \mathrm{kS} / \mathrm{s} /$ channel. PLC Siemens Simatic 1215 connected to PC used as a user interface automatically control whole procedure including fast acting valve timing. The chamber is equipped by thermocouple for temperature monitoring, especially for measurement of temperature in time of ignition. Correct hydrogen gas - air mixture composition is assured by partial pressure method. Pressure transducer measures internal pressure. For the experiment $99.995 \%$ hydrogen guaranteed by Siad, s.r.o. has been used [7].

\subsection{Experimental procedure}

Procedure for gas explosion starts with evacuation to pressure less than 0.4 bar to leave a space for the gas. For example, the use of hydrogen concentrations of $30 \mathrm{vol} \%$ and 40 vol. \% require the partial pressures of methane to be added are 0.3 bar and 0.4 bar respectively. Then the evacuation pressures should be 0.7 bar and 0.8 bar respectively. PLC starts the experiment where $60 \mathrm{~ms}$ is counted as ignition delay time, and then the gas is mixed by blowing dispersion air into the hydrogen-air mixture inside the chamber. After that, the mixture is ignited by the electric discharge. The explosion indices are measured and calculated [7].

\section{Results}

\subsection{High-speed images}

Figure 2 illustrates the real non-adiabatic explosion. The first left foto in the Figure 2 illustrates the nearly laminar spherical flame that have been produced during the first phase (i.e. in the first $10 \mathrm{~ms}$ ) of the explosion. Beyond in hundreds of ms the spherical flame became turbulent and the flame speed increases monotonically with increasing turbulences and fuel air mixing.
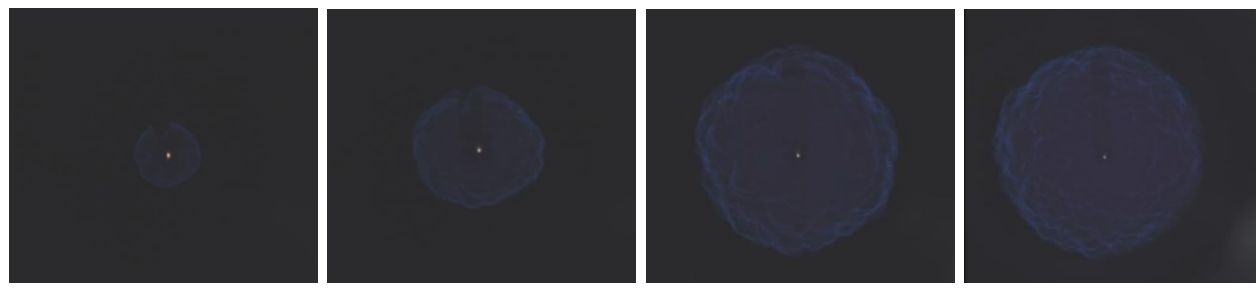

Fig. 2. High-speed images illustrating the evolution of hydrogen gas-air spherical flames.

\subsection{Explosion indices}

Figure 3 illustrates the normalized peak explosion pressure, $\mathrm{P}_{\mathrm{ex}} / \mathrm{P}_{0}$, versus equivalence ratio at atmospheric pressure and four initial temperatures. The $\mathrm{P}_{\mathrm{ex}} / \mathrm{P}_{0}$ explosion pressure is the maximum value of explosion pressure, as indicated in Figure 3. With the increase of temperature, the peak explosion pressure is decreased due to the decrease of the total fuel mass in gas/vapour cloud (turbulences). This is because the species in flames at elevated temperatures are more reactive and taking into account the effect of density (point 
concentrations). As shown in Figure $4,(\mathrm{dP} / \mathrm{dt})_{\max }$ and $\mathrm{K}_{\mathrm{G}}$ give an approximate value at varied initial temperatures, indicating that $(\mathrm{dP} / \mathrm{dt})_{\max }$ and $\mathrm{K}_{\mathrm{G}}$ are sensitive to the variation of temperature. Rate of pressure rise is affected by both flame speed (turbulences) and heat release (point concentrations). Flame speed increases monotonically with the increase of temperature, but the decrease of total fuel mass results in the decreased heat release and offsets the effect of flame speed on the explosion pressure.

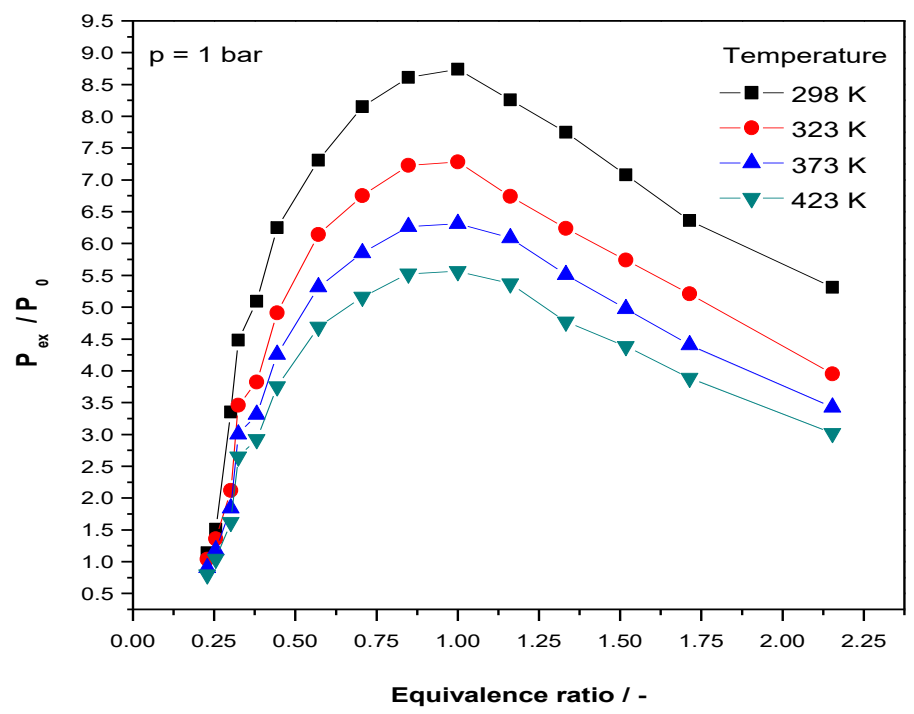

Fig. 3. Normalized peak explosion pressure, $\mathrm{P}_{\mathrm{ex}} / \mathrm{P}_{0}$, versus equivalence ratio.

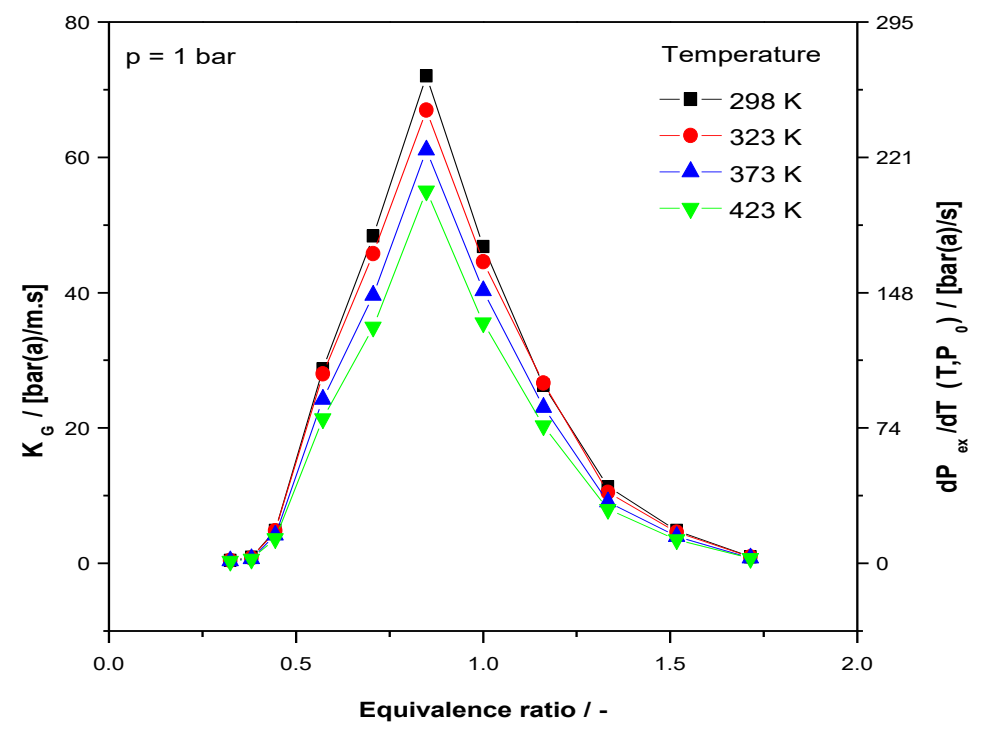

Fig. 4. Rate of pressure rise, $(\mathrm{dP} / \mathrm{dt})_{\mathrm{ex}}$, and deflagration index, $\mathrm{K}_{\mathrm{G}}$. 


\section{Conclusion}

Explosion indices data are essential for a quantitative risk assessment of explosion hazard associated with the use of hydrogen gas. The present work is the preliminary study to investigate the influence of various initial temperatures on explosion characteristics of hydrogen gas/air mixtures. The explosion pressure, the maximum rate of pressure rise and the deflagration index are the fundamental explosion characteristics. We have described, that they have decreased with the increase of initial temperature. In all cases, the turbulent gas flow and point concentrations play crucial role in the promotion of the whole reactions due to fuel air mixing and hence the faster flame speed, and heat release. In the following experiments, these values will be used as approximate initial values for explosion experiments carried out in heated $1 \mathrm{~m}^{3}$ explosion apparatus built by OZM Research s.r.o. at Energy Research Centre, VŠB - Technical University of Ostrava.

This work was prepared within the projects: 1) „Innovation for Efficiency and Environment Growth", identification code LO1403 with the financial support from the Ministry of Education, Youth and Sports in the framework of the National Sustainability Programme I and 2) Energy use by ORC technology and turbines, identification code SP2018/171.

\section{References}

1. J. Skrinsky, J. Veres, V. Peer, P. Friedel, J. Travnickova, A. Dalecka, Chemical Engineering Transactions, 53, 7-12 (2016)

2. J. Skrinsky, J. Veres, V. Peer, P. Friedel, AIP Conference Proceedings, 1745, art. no. 020057 (2016)

3. K. Hochtappels, Journal of Loss Prevention in the Process Industries, 19, 4 (2006)

4. K.L. Cashdollar, I.A. Zlochower, G.M. Green, R.A Thomas, M. Hertzberg, Journal of Loss Prevention in the Process Industries, 13, 3-5 (2000)

5. EN 1839:2013. Determination of explosion limits of gases and vapors. Berlin: Beuth Verlag (2013)

6. EN 15967:2012. Determination of maximum explosion pressure and the maximum rate of pressure rise of gases and vapours. Berlin: Beuth Verlag (2012)

7. B. Janovsky, J. Skrinsky, J. Cupak, J. Veres, Draft-paper in 12th International Symposium on Hazards, Prevention and Mitigation of Industrial Explosions Kansas City, USA - August 12-17 (2018) 